\title{
Eligibility for partial breast radiotherapy in England
}

Carolyn W Taylor FRCR, DPhil ${ }^{a, b}$, David Dodwell FRCR, MD ${ }^{a, b}$,

Sarah C Darby FMedSci, FRS ${ }^{a}$, John Broggio MScic ${ }^{c}$, Paul McGale PhD ${ }^{a}$

${ }^{a}$ Nuffield Department of Population Health, University of Oxford, UK

${ }^{b}$ Oxford University Hospitals NHS Foundation Trust, Oxford, UK

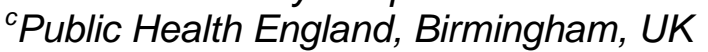

${ }^{*}$ Corresponding author:

Carolyn Taylor

Nuffield Department of Population Health,

University of Oxford,

Richard Doll Building,

Old Road Campus, Oxford

OX3 7LF

United Kingdom

Email: carolyn.taylor@ndph.ox.ac.uk

Declarations of interest: none

Words: $<1,500$ excluding references and title page

Figure: 1

Supplementary figure: 1 
Randomised trials involving a total of more than 10,000 women have confirmed that radiotherapy to the breast reduces the risks of cancer recurrence and of death after breast conserving surgery [1]. Where reported in these trials, the main site of local recurrence was the same area as that of the index cancer, i.e. the tumour bed $[2,3]$. Therefore, irradiating just the tumour bed with a margin, as in partial breast radiotherapy, may maintain the benefits of radiotherapy but avoid the side effects of irradiating the whole breast. In the UK IMPORT LOW trial women with low risk breast cancer were randomised to partial breast versus whole breast intensity modulated radiotherapy, and it was found that partial breast was as effective as whole breast radiotherapy in reducing cancer recurrence [4]. Partial breast radiotherapy may involve a variety of techniques, including external beam radiotherapy, brachytherapy and intra-operative radiotherapy. The effects of these different types of partial breast radiotherapy will be assessed in a meta-analysis of all available trials of partial breast radiotherapy, regardless of technique, by the Early Breast Cancer Trialists' Collaborative Group.

Partial breast radiotherapy is currently recommended in the UK for women who have breast cancer with low risk of recurrence. The National Institute of Clinical Excellence (NICE) recommends consideration of partial breast radiotherapy in women who are aged $\geq 50$ years old, have received breast conserving surgery for tumours of $\leq 3 \mathrm{~cm}$ that are node negative, oestrogen receptor positive, HER2 receptor negative, and are grades 1 or 2 [5]. They also suggest consideration of omission of radiotherapy for a subset of these women who are $\geq 65$ years old with clear pathological margins following surgery, after discussion of the benefits and risks of radiotherapy with the patient. The Royal College of Radiologists' (RCR) consensus guidelines $[6,7]$ are slightly more stringent and, in addition, they stipulate that partial breast radiotherapy should be considered only for tumours that have a radial excision margin of at least $2 \mathrm{~mm}$ provided they are not lobular and do not have lymphovascular invasion. 
Estimating the percentage of women irradiated for breast cancer who would be eligible for partial breast radiotherapy is informative for two reasons. First, it provides a baseline figure against which national audits can be compared to assess how widely evidence from partial breast radiotherapy trials has been implemented. Second, it informs radiotherapy resource allocation at a radiotherapy centre level. Omission of radiotherapy saves resources and partial breast radiotherapy may allow shorter radiotherapy schedules thus reducing resource needs and costs compared with whole breast radiotherapy.

We used data from women diagnosed with breast cancer in England during 2012-2016 to estimate percentages of women who would be eligible for partial breast radiotherapy according to both the NICE guidelines and the RCR consensus in all women who received adjuvant breast cancer radiotherapy in England over a 5-year period.

We based percentages on the number of women who had surgery and radiotherapy for their breast cancer. A total of 198,059 women in England were recorded by the National Cancer Registration and Analysis System as having a diagnosis of breast cancer (Figure 1). From these, 2,589 were excluded on the basis of their histology (e.g. sarcoma or non-invasive). A further 62,140 women were excluded because follow-up variables indicated that their original treatment intent was palliative or because it was not possible to confirm the original treatment intent or because they received neoadjuvant chemotherapy or because they did not have breast surgery. Of the remaining 133,330 women who received surgery for early breast cancer, 99,501 women were recorded as receiving breast cancer radiotherapy and for 67,878 of them there was information on the required variables for partial breast radiotherapy guidelines. In these women, 24,522/67,878 (36\%) women met NICE criteria for partial breast radiotherapy and $21,388 / 67,878$ (32\%) met the RCR criteria. For the RCR criteria, women with lymphovascular invasion or an excision margin $<2 \mathrm{~mm}$ could not be excluded because information on these variables was unavailable for most women. Therefore the actual percentage of women eligible for partial breast radiotherapy will be 
lower than $32 \%$. Published data can be used to estimate the numbers of additional women who may have these factors in our data. In the largest partial breast radiotherapy trial to date (TARGIT A) $13 \%$ of eligible women had lymphovascular invasion [8]. If $13 \%$ of the 21,388 women otherwise eligible for partial breast radiotherapy according to the RCR consensus had lymphovascular invasion, 2780 additional women would be ineligible, and the overall percent eligible would reduce to $27 \%$. This percentage would reduce further if women with $<2 \mathrm{~mm}$ radial margin were removed. Calculations based on women diagnosed with stages 1 or 2 breast cancer (rather than women who received radiotherapy) are found in Figure S1.

\section{International guidelines}

Partial breast radiotherapy guidelines vary at present. The European Society for Radiotherapy and Oncology (ESTRO) guidelines [9] are similar to UK guidelines, except that eligible patients may have tumours that are high grade or oestrogen receptor negative. ESTRO also defines an intermediate-risk group who may receive partial breast RT within a clinical study. The American Society for Radiation Oncology (ASTRO) defines three patient groups: suitable, cautionary and unsuitable [10]. Most patient groups recommended for consideration of partial breast radiotherapy in the UK guidelines would be included within the ASTRO suitable and cautionary groups.

In the UK, it is unknown whether use of partial breast radiotherapy is as high as our suggested percentages above. However, it has increased during the past two decades. Before the year 2000, partial breast radiotherapy was not routinely recommended. During 2000 to 2012 many patients eligible for partial breast radiotherapy were randomised within the IMPORT low and TARGIT A trials, and around half of them would have received partial breast radiotherapy. Results of IMPORT low were first reported in abstract form during 2016 [11], and later that year the RCR defined a group suitable for partial breast RT outside the trial setting. It is likely that since 2016, an increasing number of patients in the UK have been considered for partial breast radiotherapy. The extent to which this has occurred may be 
addressed by future breast radiotherapy audits. Radiotherapy omission is now considered for women with low risk breast cancer within the UK PRIMETIME study [12]. The results of this study may affect guidelines and so in the future, the number of women eligible for partial breast radiotherapy may actually reduce. These changes are likely to reduce side-effects and pressure on radiotherapy centres whilst retaining patient benefits.

Acknowledgements: This work was funded by a research contract to the University of Oxford under the Department of Health Policy Research Programme (Studies of lonising Radiation and the Risk of Heart Disease). CT, PMG and SD received funding from Cancer Research UK (grant C8225/A21133).

We would like to thank Jackie Charman for her work on the Public Health England data.

Procedures for accessing the data for this study are available on: https://www.ndph.ox.ac.uk/files/about/ndph-data-access-policy-1.pdf 


\section{References}

1 Early Breast Cancer Trialists' Collaborative Group. Effect of radiotherapy after breastconserving surgery on 10-year recurrence and 15-year breast cancer death: meta-analysis of individual patient data for 10801 women in 17 randomised trials. Lancet 2011;378:17071716.

2 Fisher ER, Sass R, Fisher B, Gregorio R, Brown R, Wickerham L, Pathologic findings from the National Surgical Adjuvant Breast Project (protocol 6). II. Relation of local breast recurrence to multicentricity. Cancer 1986;57:1717-24.

3 Veronesi U, Luini A, Del Vecchio M, Greco M, Galimberti V, Merson M, et al. Radiotherapy after breast-preserving surgery in women with localized cancer of the breast. New Eng $\mathrm{J}$ Med1993;328:1587-91.

4 Coles C, Griffin CL, Kirby AM, Titley J, Agrawal RK, Alhasso A, et al. Partial breast radiotherapy after breast conservation surgery for patients with early breast cancer (UK IMPORT LOW trial): 5 year results from a multicentre, randomised, controlled, phase 3, non-inferiority trial. Lancet 2017;390:1048-60.

5 National Institute for Health and Care Excellence (Last updated: July 2018) Early and locally advanced breast cancer: diagnosis and treatment (NICE Clinical Guideline NG101). Available at: https://www.nice.org.uk/guidance/ng101/chapter/Recommendations\#radiotherapy [Accessed 30 March 2019].

6 The Royal College of Radiologists. Postoperative radiotherapy for breast cancer: UK consensus statements. London: The Royal College of Radiologists, 2016.

7 Bloomfield DJ, on behalf of the Core Group facilitated by the Royal College of Radiologists. Development of postoperative radiotherapy for breast cancer: UK consensus statements a model of patient, clinical and commissioner engagement? Clin Oncol 2017; 29: 639-41.

8 Vaidya JS, Joseph DJ, Tobias JS, Bulsara M, Wenz F, Saunders C, et al. Targeted intraoperative radiotherapy versus whole breast radiotherapy for breast cancer (TARGIT-A trial): an international, prospective, randomised, non-inferiority phase 3 trial. Lancet 2010;376:91-102.

9 Polgar C, Van Limbergen E, Potter R, Kovacs G, Polo A, Lyczek J, et al. Patient selection for accelerated partial-breast irradiation (ABPI) after breast conserving surgery:

Recommendations for the Groupe Europeen de Curietherapie-European Society for Therapeutic Radiology and Oncology (GEC-ESTRO) breast cancer working group based on clinical evidence. Radioth Oncol 2010;94:264-273.

10 Correa C, Harris EE, Leonardi MC, Smith BD, Taghian AG, Thompson AM, et al. Accelerated partial breast irradiation: Executive summary for the update of an ASTRO evidence-based consensus statement. Pract Radiat Oncol 2017;7:73-79.

11 Coles C, Agrawal R, Ah-See ML, Algurafi H, Alhasso A, Brunt AM, et al. Partial breast radiotherapy for women with early breast cancer: first results of local recurrence data for IMPORT LOW (CRUK/06/003). Late breaking abstract presentation at EBCC, 09/03/2016, Amsterdam.

12 Kirwan CC, Coles CE, Bliss J; the PRIMETIME Protocol Working Group. It's PRIMETIME. Postoperative avoidance of radiotherapy: Biomarker selection of women at very low risk of 
local recurrence. Clin Oncol 2016; 28 :594-6. 


\section{Figure legends}

Figure: Women who received adjuvant radiotherapy, among women diagnosed with breast cancer in England during January 2012-December 2016. Number and percent eligible for partial breast radiotherapy according to UK guidelines

Footnotes:

*Women whose treatment intent was probably palliative were identified as follows: record of drug usually given for metastatic disease, record of palliative chemotherapy or palliative radiotherapy within a year of breast cancer diagnosis, record of metastatic disease or second cancer or death within 3 months of breast cancer diagnosis.

† Most women with no follow-up had their cancer registered on their date of death.

¥ Women who received neoadjuvant chemotherapy were excluded because their tumour information may have been obtained after chemotherapy.

${ }^{* *}$ Consensus also specified radial excision margin $\geq 2 \mathrm{~mm}$ and no lymphovascular invasion. These variables were unavailable in the data so were not included in calculations

Abbreviations:

NICE: National Institute of Clinical Excellence

RCR: Royal College of Radiologists

Figure S1. Women with stages 1 or 2 breast cancer, among women diagnosed with breast cancer in England during January 2012-December 2016. Number and percent eligible for partial breast radiotherapy according to UK guidelines

Footnotes:

*Women whose treatment intent was probably palliative were identified as follows: record of drug usually given for metastatic disease, record of palliative chemotherapy or palliative radiotherapy within a year of breast cancer diagnosis, record of metastatic disease or second cancer or death within 3 months of breast cancer diagnosis.

$\dagger$ Most women with no follow-up had their cancer registered on their date of death.

¥ Women who received neoadjuvant chemotherapy were excluded because their tumour information may have been obtained after chemotherapy.

${ }^{* *}$ Consensus also specified radial excision margin $\geq 2 \mathrm{~mm}$ and no lymphovascular invasion. These variables were unavailable in the data so were not included in calculations

Abbreviations:

NICE: National Institute of Clinical Excellence

RCR: Royal College of Radiologists 
Women diagnosed with breast cancer in

England during 1.1.2012-31.12.2016

$\mathrm{N}=198,059$

\section{Exclusions}

\section{Inappropriate histology $\mathbf{N}=2,589$}

- Non-breast e.g. sarcoma, $\mathrm{N}=1,728$

- Non-invasive e.g. carcinoma in situ or microinvasion only $\mathrm{N}=861$

\section{Treatment intent palliative/unknown or} neoadjuvant therapy given or no surgery $\mathrm{N}=62,140$

- Metastatic or second primary cancer likely* $N=26,009$

- No follow-upt $\mathrm{N}=3,016$

- Received neoadjuvant chemotherapy $¥ \mathrm{~N}=16,367$

- No record of breast surgery $\mathrm{N}=16,748$
Women who had surgery for early

breast cancer

$\mathrm{N}=133,330$
No record of radiotherapy $\mathrm{N}=33,829$

Women who received adjuvant breast cancer radiotherapy

$\mathrm{N}=99,501$

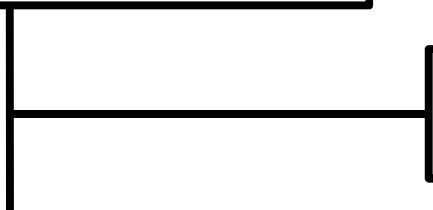

Full data unavailable $\mathrm{N}=31,623$

Total women with full data $\mathrm{N}=67,878$

Number meeting NICE criteria: 24,522/67,878 (36\%)

Women aged $\geq 50$ years, received breast conserving surgery, tumour size $\leq 3 \mathrm{~cm}$, node negative, oestrogen receptor positive, HER2 receptor negative, grade 1 or 2 .

Number meeting RCR criteria: $21,388 / 67,878$ (32\%)

As above but also not lobular cancer** 
Women diagnosed with breast cancer in

England during 1.1.2012-31.12.2016

$\mathrm{N}=198,059$

\section{Exclusions}

\section{Inappropriate histology $\mathbf{N}=\mathbf{2 , 5 8 9}$}

- Non-breast e.g. sarcoma, $\mathrm{N}=1,728$

- Non-invasive e.g. carcinoma in situ or microinvasion only $\mathrm{N}=861$

\section{Treatment intent palliative/unknown} or neoadjuvant therapy given $\mathrm{N}=45,392$

- Metastatic or second primary cancer likely* $N=26,009$

- No follow-upt $\mathrm{N}=3,016$

- Received neoadjuvant chemotherapy $¥ \mathrm{~N}=16,367$

Women with early breast cancer

$\mathrm{N}=150,078$

Cancers NOT Stage I or II $\mathrm{N}=25,649$

Women with Stages 1 or 2 breast cancer

$\mathrm{N}=124,429$

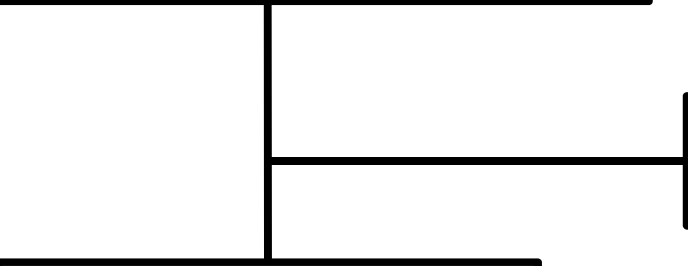

Full data unavailable $\mathrm{N}=46,133$

Total women with full data

$\mathrm{N}=78,296$

Number meeting NICE criteria: : 26,155/78,296 (33\%)

Women aged $\geq 50$ years, received breast conserving surgery, tumour

size $\leq 3 \mathrm{~cm}$, node negative, oestrogen receptor positive,

HER2 receptor negative, grade 1 or 2 .

\section{Number meeting RCR criteria: 22,834/78,296 (29\%)}

As above but also not lobular cancer** 